Radial and Nonradial Pulsations as Prabes of Stellar Physics

ASP Conference Series, Vol. 259, 2002

C. Aerts, T.R. Bedding, \& J. Christensen-Dalsgaard, eds.

\title{
Tuning Pulsation Modes with Stellar Interferometry
}

\author{
A. Domiciano de Souza Jr. ${ }^{1}$, F. Vakili, S. Jankov ${ }^{2}$ \\ Observatoire de la Côte d'Azur, France \\ E. Janot-Pacheco \\ Instituto Astronômico e Geofísico - USP, São Paulo, SP, Brazil
}

\begin{abstract}
Stellar interferometry represents a qualitative jump toward stellar surface imaging. We propose a method that tunes and filters out high degree non-radial pulsation (NRP) modes from the Fourier plane associated to the stellar brightness distribution. The proposed method can be applied to continuum flux, slow stellar rotation and high degree multi mode NRP stars.
\end{abstract}

\section{Introduction}

Stellar interferometry explores the Fourier $u v$ plane of sky projected brightness distributions. If a good coverage of this plane is available (several apertures) a particular brightness pattern on the stellar surface can be isolated by applying a judiciously chosen spatial filter. In the case of NRP one can associate a spatial filter to each pulsation mode. A recent example of interferometric techniques applied to the study of NRP can be found in Jankov et al. (2001).

The codes TLUSTY and SYNSPEC (Hubeny \& Lanz, 1995) were used to synthesize the photospheric local intensities. Townsend's NRP model (Townsend, 1997) has been adapted in order to calculate the monochromatic intensity stellar maps. These models have been included in an interferometric-oriented numerical code to study the performances of the spatial filtering.

\section{Results and conclusions}

In the present paper we only consider the squared Fourier maps (visibility $V$ maps), which are most commonly measured by stellar interferometers. Fig. 1 illustrates the application of the spatial filtering to a high degree multi mode NRP star. The effects of the pulsation modes on the visibility maps are more clearly seen by considering the visibility differences $\Delta V\left(=V-V_{\text {ref }}\right.$; where $V_{\text {ref }}$ is the visibility map for a non-pulsating homogenous star).

\footnotetext{
${ }^{1}$ e-mail: Armando.Domiciano@obs-azur.fr

${ }^{2}$ Present address: Astronomical Observatory Beograd, Beograd, Yugoslavia
} 

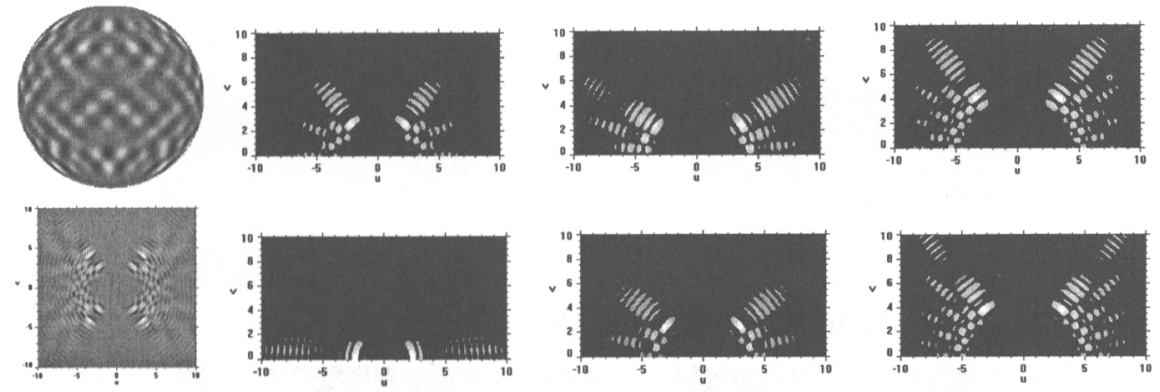

Figure 1. Spatial filtering applied to a star with selected NRP modes $(\ell, m)=(20,-12),(25,20)$ and $(30,-18)$. The brightness distribution and visibility difference $\Delta V$ maps are shown top and bottom left, respectively. Calculations were performed in the adjacent continuum of HeI6678. The six images to the right are the spatial filters obtained from the theoretical $\Delta V$ maps of the same NRP modes and phases used to model the star (upper row) and of three other modes and phases (lower row) with $(\ell, m)=(12,-12),(23,17)$ and $(30,-18)$. Only half of the $u v$ coverage is shown due to symmetry properties. The filters have 10 transmission levels, which are proportional to the intensities in the $\Delta V$ maps. The correlation factors are at least 3 times higher for the correct filters (upper row) than for the other ones (lower row).

The presence of multi modes on the star introduces a complex pattern in the corresponding $u v$ plane. However, by testing different spatial filters defined by different NRP modes, it is possible to identify which modes are present in the star. The quality of a spatial filter is evaluated by the integral of the product of $\Delta V$ by the filter. The result is a number (called correlation factor) that measures the possibility that a given NRP mode with a given phase is present on the star. For the examples chosen here the correlation factors are at least 3 times higher for the correct filters compared to the wrong ones. Note that even the correct phases of the oscillations can be recovered by this method.

The proposed method can be applied to continuum flux, slow stellar rotation and high-degree multi-mode NRP stars. A deeper study is necessary in order to better evaluate the performances of the method. Future space-based multiaperture interferometers will attain spatial frequencies high enough to allow the application of spatial filtering.

\section{References}

Hubeny, I. \& Lanz, T. 1995, ApJ, 439, 875

Jankov, S., Vakili, F., Domiciano, A.D.Jr., \& Janot-Pacheco, E. 2001, A\&A, in press

Townsend, R.H.D. 1997, MNRAS, 284, 839 